\title{
OPTIMIZING OPERATIONS OF LARGE WATER SUPPLY NETWORKS A Case Study
}

\author{
Derek Verleye, El-Houssaine Aghezzaf and Dimi Defillet \\ Department of Industrial Engineering, Faculty of Engineering and Architecture, Ghent University, \\ Technologiepark 903, Zwijnaarde, Belgium
}

Keywords: Drinking water supply, Mixed integer nonlinear programming, Network optimization.

\begin{abstract}
In this paper we propose a mathematical programming model for a large drinking water supply network and discuss some possible extensions. The proposed optimization model is of a real water distribution network, the largest water supply network in Flanders. The problem is nonlinear, nonconvex and involves some binary variables, making it belong to the class of NP-hard problems. We discuss a way to convexify the nonconvex term and show some results on two case instances of the actual network.
\end{abstract}

\section{INTRODUCTION}

Optimization of drinking water networks has recently regained attention of many researchers in mathematical programming. These models are typically nonconvex MINLP's and are in general hard to solve. They are often solved by means of some NLP solvers, after the binary variables are relaxed or the model reformulated (Cembrano et al., 2000; Burgschweiger et al., 2009). Heuristic methods are also frequently used to solve these models (Savic and Walters, 1997; López-Ibáñez et al., 2008; Nicklow et al., 2010).

In this paper, we extend the model used to optimize an existing network managed by a company in Flanders, Belgium (Verleye and Aghezzaf, 2011). This extended model is a nonconvex MINLP for which we linearize the power term. It is then solved by means of an open-source solver and tested on two recent cases (Defillet, 2011).

\section{MODEL FORMULATION}

Before discussing the solution approach, we first provide an overview of the model. The components and restrictions discussed here are based on an existing supply network of a large drinking water supplier in Flanders, Belgium. We model the network using a directed graph $\mathcal{G}=(\mathcal{N}, \mathcal{A})$, where $\mathcal{N}$ is the node set representing junctions $(\mathcal{J})$, delivery points $(\mathcal{D})$, buffers $(\mathcal{B})$ and raw water sources $(\mathcal{S})$; and $\mathcal{A}$ is the arc set representing pipes $(\mathcal{P} i)$, pipes with pure water pumps $(\mathscr{P} u)$ and pipes with raw water pumps $(\mathcal{R})$. Furthermore, a day is divided in periods $t \in[1, T]$, where $T$ represents the number of periods. The length of each period is denoted by $\tau_{t}$.

The main variables in a water supply network are the flow values, $Q_{t}^{i j}$ on arc $(i, j)$, and the piezometric heads in each node $i, H_{t}^{i}$. Piezometric head is the sum of the static level $\left(h^{i}\right)$ and the manometric pressure. Since time is discretized, we interpret the variables as follows. The flows are assumed to be constant during the interval $[t-1, t]$. The head values will be interpreted as values at time $t$. The volume in a tank, $V_{t}^{i}$, is the available volume at the end of period $t$.

In our model, the amount to be produced during each period needs to be determined. Moreover, at each time period we need to know which pumps to activate for the period to come and at which frequency.

\subsection{Restrictions on Nodes $(i \in \mathcal{N})$}

On nodes, some bounds on manometric pressure apply. Conservation of flow is written for each junction. In what follows, we describe constraints on buffers.

\subsubsection{Buffers $(i \in \mathcal{B})$}

We consider buffers with free inflow $\left(I_{t}^{i}(+)\right)$ in the tank, whereas outflow is possible through high pressure because of the tank level $\left(I_{t}^{i}(-)\right)$ or by pumping 
water out of the buffer $\left(O_{t}^{i}(-)\right)$. For more details, we refer the reader to (Verleye and Aghezzaf, 2011).

Flow conservation:

$$
\begin{aligned}
\sum_{k:(k, i) \in \mathcal{A}} Q_{t}^{k i}-\sum_{j:(i, j) \in \mathcal{A} \backslash \mathcal{P} u} Q_{t}^{i j} & =I_{t}^{i}(+)-I_{t}^{i}(-) \\
\sum_{j:(i, j) \in \mathcal{P}_{u}} Q_{t}^{i j} & =O_{t}^{i}(-)
\end{aligned}
$$

Tank volume:

$$
\begin{aligned}
& V_{t}^{i}=V_{t-1}^{i}+\left(I_{t}^{i}(+)-I_{t}^{i}(-)-O_{t}^{i}-d_{t}^{i}\right) \tau_{t} \\
& V_{0}^{i} \geq V_{T}^{i}
\end{aligned}
$$

$d_{t}^{i}$ denotes demand.

Mean piezometric level $\left(M_{t}^{i}\right)$ in tank:

$$
M_{t}^{i}=l f^{i}+\frac{L_{t}^{i}+L_{t-1}^{i}}{2}
$$

where $L_{t}^{i}=\frac{V_{t}^{i}}{A^{i}}$ is the (approximated) water level in the tank, with $A^{i}$ the cross-sectional area of tank.

Tank in- or outflow:

$$
\begin{aligned}
I_{t}^{i}(+) & \leq A^{i} l^{i}(\max ) X_{t}^{i} \\
I_{t}^{i}(-) & \leq A^{i} l^{i}(\max ) Y_{t}^{i} \\
H_{t}^{i}-l i^{i} & \geq-100\left(1-X_{t}^{i}\right) \\
M_{t}^{i}-H_{t}^{i} & \geq-100\left(1-Y_{t}^{i}\right)
\end{aligned}
$$

Here, $l i^{i}$ is the level at which water flows (freely) in the tank. The binary variables $X_{t}^{i}, Y_{t}^{i}$ are used to model in- and outflow. If $I_{t}^{i}(+)>0$ pressure $H_{t}^{i}$ at the entrance has to be higher than the inflow level $l i{ }^{i}$. In the same way, outflow can only occur if the water level is sufficiently high. The number 100 is derived from the fact that the manometric pressure cannot exceed $10 \mathrm{bar}(100 \mathrm{~m})$ in any part of the network.

\subsection{Restrictions on $\operatorname{Arcs}((i, j) \in \mathcal{A})$}

\subsubsection{Pipes $((i, j) \in \mathcal{P} i)$}

Flow bounds ((Bragalli et al., 2006)):

$$
-3600 \frac{\pi}{4} v_{\max }^{i j}\left(d^{i j}\right)^{2} \leq Q_{t}^{i j} \leq 3600 \frac{\pi}{4} v_{\max }^{i j}\left(d^{i j}\right)^{2}
$$

$d^{i j}$ is the diameter and $v_{\max }^{i j}$ the maximum flow speed. Pressure losses:

$$
H_{t}^{i}-H_{t}^{j}=\kappa^{i j} Q_{t}^{i j}\left|Q_{t}^{i j}\right|
$$

which is the formula of Darcy-Weisbach and is suitable for turbulent flow in pipes. The loss coefficient $\kappa^{i j}$ depends on pipe length, diameter and the pipe friction coefficient. To determine the last one, we work with the (simplified) law of Prandtl-Kármán for hydraulically rough pipes.

\subsubsection{Pure Water Pumps $((i, j) \in \mathcal{P} u)$}

The following constraints apply on constant speed pumps. In addition to flow bounds, we can again write the pressure loss constraints which are slightly different here because water can flow only in one direction and the pump increases the head, $\Delta H_{t}^{i j}$ :

Pressure losses:

$$
\begin{array}{ll}
H_{t}^{i}-H_{t}^{j}-\kappa^{i j}\left(Q_{t}^{i j}\right)^{2}+\Delta H_{t}^{i j}=0 & \forall i \in \mathcal{N} \backslash \mathcal{B} \\
M_{t}^{i}-H_{t}^{j}-\kappa^{i j}\left(Q_{t}^{i j}\right)^{2}+\Delta H_{t}^{i j}=0 & \forall i \in \mathcal{B}
\end{array}
$$

The second constraints apply to pumps extracting water from a tank with level $M_{t}^{i}$.

Pump characteristic:

Using data provided by pump suppliers we approximate the relation between head, $\Delta H$, and flow, $Q$ as

$$
\Delta H_{t}^{i j}=h_{1}^{i j}\left(Q_{t}^{i j}\right)^{2}+h_{2}^{i j} Q_{t}^{i j}+h_{3}^{i j}
$$

where $h$ are the function coefficients. In the same way, the efficiency $E$ is given by

$$
E_{t}^{i j} \leq e_{1}^{i j}\left(Q_{t}^{i j}\right)^{2}+e_{2}^{i j} Q_{t}^{i j}+e_{3}^{i j}
$$

with coefficients $e$. We can relax the equality constraint because efficiency will always be chosen to be as high as possible. The power term $P$ is given by

$$
P_{t}^{i j}=2,73 \frac{\Delta H_{t}^{i j} Q_{t}^{i j}}{E_{t}^{i j}}
$$

To avoid numerical difficulties, we force $e_{3}^{i j}>0$ so to prevent the efficiency from becoming 0 when the pump is not working, that is when $Q_{t}^{i j}=0$. This will prevent the denominator of the power term, $P$, from becoming 0 .

Substituting the approximations for efficiency and head in (16), we can also draw a curve for the power based on pump data (see fig 1). We can then approximate (16) with:

$$
P_{t}^{i j}=p_{1}^{i j} Q_{t}^{i j}+p_{2}^{i j}
$$

\subsubsection{Raw Water Pumps $((i, j) \in \mathcal{R})$}

In addition to treatment capacity and daily extraction limits in ground water treatments, we include bounds on subsequent production flow rates:

$$
\begin{gathered}
-q^{i j}(\text { fluct }) \leq Q_{t}^{i j}-Q_{t-1}^{i j} \leq q^{i j}(\text { fluct }) \\
-2 q^{i j}(\text { fluct }) \leq Q_{T}^{i j}-Q_{0}^{i j} \leq 2 q^{i j}(\text { fluct })
\end{gathered}
$$

These bounds will prevent quality deterioration due to excessive changing of production amount from one period to the next. 


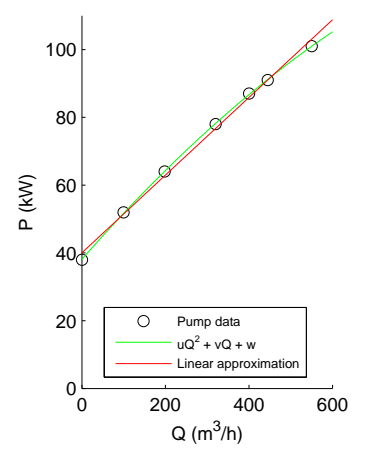

Figure 1: Fitted power function using pump data.

\subsection{Models}

As two different cases will be tested, the models will be slightly different. The preceding restrictions are applicable for both models. In what follows additional restrictions for each model will be described.

\subsubsection{Decision Support Model for the Region West-Flanders}

Due to a recent network expansion, the question on how to optimally satisfy the demand by using existing sources arises.

The function that needs to be minimized is the total cost, consisting of energy delivered by the pumps and production/electricity at the water production centers:

$$
\operatorname{Min} \sum_{t=1}^{T}\left(\sum_{(i, j) \in \mathcal{P}_{u}} P_{t}^{i j} \frac{c_{t}(e)}{1000}+\sum_{(i, j) \in \mathcal{R}} Q_{t}^{i j} c^{i j}(p)\right) \tau_{t}
$$

\subsubsection{High-level Transport Optimization Model}

Due to the recent collaboration of different drinking water companies, a significant extra amount of drinking water will be flowing through the main supply network. Here arises the need of a high level decision model that investigates the impact of these changes on the operation of the drinking water supply network.

The objective function (20) is extended with

$$
\sum_{t=1}^{T}\left(\sum_{j:(i, j) \in \mathcal{A}} Q_{t}^{i, j}-\sum_{k:(k, i) \in \mathcal{A}} Q_{t}^{k, i}\right) a p^{i} \tau_{t} \quad \forall i \in \mathcal{D}
$$

where $a p^{i}$ denotes the price (in $e / m^{3}$ ) for delivering water to node $i$.

At the ground water sources in this region, raw water pumps work individually and are set to a constant flowrate. Furthermore, switching these pumps on/off shouldn't occur frequently. For each group of raw water pumps we define the set $\mathcal{C}$ which contains all possible combinations of discrete total flowrates. We avoid adding binary variables and let $\alpha^{m(i j)}$ be 1 when the raw water pumps at $i j$ operate at combination $m$. Then (for $0 \leq \alpha \leq 1$ ):

$$
Q_{t}^{i j}=\sum_{m \in C(i j)}\left(\alpha^{m} b c^{i, j}\right) \quad \forall(i, j) \in \mathcal{R}
$$

where $b c^{i, j}$ is the treatment capacity. As a consequence, the amount of pumped water remains constant over time. Of course, only one possible combination can be chosen:

$$
\begin{array}{rr}
\sum_{m \in C(i j)} \alpha^{m} & \geq 1 \\
\alpha^{m} \alpha^{n} & \leq \text { tol } \quad \forall(i, j) \in \mathcal{R} \\
& \forall m, n \in C(i j):(i, j) \in \mathcal{R}
\end{array}
$$

where $t o l$ is a very small value.

\section{COMPUTATIONAL RESULTS}

In this section we discuss the most important results obtained from the two proposed models. Both models, were implemented in AMPL (Fourer et al., 2003) where the open-source solver BONMIN (Bonami and Lee, 2006) was used to generate solutions. This solver, suitable only for smooth and convex MINLP, will only provide local solutions for our nonconvex models. In both cases we encountered some numerical difficulties due to buffer in and outflow constraints (6-9). We relax these constraints and allow only inflow in buffers. This is not a major issue, however the solution value may be slightly inferior because we don't admit outflow.

\subsection{Decision Support Model for the Region West-Flanders}

This large supply network consists of 160 nodes and 190 arcs. For a division of 1 day in 5 intervals, we find an optimal solution after 56 seconds. For a more accurate division in 24 periods of one hour, a solution is found in 642 seconds. Although this second approach is more accurate, the goal function changed only marginally. The most important results for both simulations are: First, water production centers that have a high production cost (e.g. due to ground water taxes) produce considerably less than low-cost production centers. Second, the level of water in pure water tanks remains as high as possible throughout 
the optimization period. A possible explanation for this phenomenon is the fact that the connected pure water pumps have to deliver less pressure, which results in a lower energy cost. Third, the level of the water in the buffers moves in opposite direction to the energy tariff during each period. This is a direct consequence of the fact that the pumps will mainly work during periods where the electricity tariff is low.

\subsection{High-level Transport Optimization Model}

Setting the Tolerance Value. As stated before, the value of the actual production flow in a groundwater production center is allowed to vary slightly according to a tolerance value, tol. For smaller values of $t o l$, the flow will be accurate but computation time will rise. It is observed that setting this value to 0,0001 in (24) results in a reasonable computation time and a realistic approximation of the production flow.

Water Hammer Considerations. Initial test runs showed that the flow in a particular pipe suddenly changed direction during one period and then changed again in the subsequent period. Because we want to minimize chances on water hammer, we add the additional constraint that flow can only go in the same direction for all periods. Ongoing research is conducted to prevent unwanted direction changes in all pipes during one or several periods.

Before the collaboration took place, network results are: (1) All pumps have activity points at values which indicate high levels of efficiency $(60-80 \%)$. (2) In one of the pipe sections, water has to travel more than $15 \mathrm{~km}$ before it reaches the other end. Simulation shows however that this only happens after more than 24 hours. Since no demand takes place at this pipe section, water will remain inside for quite some time, which might lead to quality issues. This is certainly something to take into consideration in future steps.

In the future collaboration, an additional $4000 \mathrm{~m}^{3}$ of water will be transferred through this part of the network. This results in: (1) additional costs. This is of course expected, but it is worth remarking that the extra cost is only due to increased electricity costs for the pure water pumps while production cost slightly drops. In one of the pump station the electricity cost is increased by as much as $90 \%$. (2) The equilibrium in the long pipe has shifted positively because of the injection of extra water in the network. The quality issue has thus decreased in size, which is a positive side effect of the collaboration. Of course we will still have to take this issue into account in other situations or for other parts of the network.

\section{CONCLUSIONS}

In this paper a drinking water network in Flanders involving constant speed pumps and buffers with free inflow is modeled. Binary variables, used to model buffer in and outflows, together with nonsmooth and nonconvex character of some constraints and the power term in the objective function complicate the model. A linear approximation of the power term was proposed, and the resulting model was solved using the open-source solver BONMIN. The model provided some initial good results on two real-life cases. Some future improvements concern the issue of preventing quality deterioration with changing directions of water flow. The unwanted water hammer effects need also to be tackled.

\section{REFERENCES}

Bonami, P. and Lee, J. (2006). Bonmin users manual. Technical report, Coin-Or.

Bragalli, C., D’Ambrosio, C., Lee, J., Lodi, A., and Toth, P. (2006). An minlp solution method for a water network problem. In $L N C S$, volume 4168, pages 696707. Springer-Verlag Berlin Heidelberg.

Burgschweiger, J., Gnädig, B., and Steinbach, M. C. (2009). Nonlinear programming techniques for operative planning in large drinking water networks. Open Appl Math J, 3:14-28.

Cembrano, G., Wells, G., Quevedo, J., Pehrez, R., and Argelaguet, R. (2000). Optimal control of a water distribution network in a supervisory control system. Control Engineering Practice.

Defillet, D. (2011). Optimalisatie van het transport van drinkwater op het niveau van het hoofdverdeelnet van de vlaamse maatschappij voor watervoorziening. Master thesis, University of Ghent.

Fourer, R., Gay, D., and Kernighan, B. (2003). AMPL: A Modeling Language for Mathematical Programming. Duxbury Press/Brooks/Cole Publishing Co.

López-Ibáñez, M., Prasad, T. D., and Paechter, B. (2008). Ant colony optimization for optimal control of pumps in water distribution networks. J Water Resour Plan Manage, 134(4):337346.

Nicklow, J., Reed, P., Savic, D., Dessalegne, T., Harrell, L., Chan-Hilton, A., Karamouz, M., Minsker, B., Ostfeld, A., Singh, A., and Zechman, E. (2010). State of the art for genetic algorithms and beyond in water resources planning and management. J Water Resour Plan Manage, 136(4):412-432.

Savic, D. A. and Walters, G. A. (1997). Genetic algorithms for least-cost design of water distribution networks. $J$ Water Resour Plan Manage, 123(2):67-77.

Verleye, D. and Aghezzaf, E.-H. (2011). Modeling and optimizing production and distribution of drinking water at vmw. In $L N C S$, volume 6701, pages 315-326. Springer-Verlag Berlin Heidelberg. 